\title{
RADIATYYE EDGES UNDER CONTROL BY IMPURITY FLUXES
}

\author{
U. Samm, G. Bertschinger, P. Bogen, J.D. Hey, E. Hintz, L. Könen, Y.T. Lie, \\ A. Pospieszczyk, D. Rusbüldt, R.P. Schorn, B. Schweer, M. Tokar', B. Unterberg \\ Forschungszentrum Jülich, Association EURATOM-KFA, Germany
}

\begin{abstract}
Experimental results from TEXTOR are presented to provide strong evidence for the feasibility of the "cold radiative plasma mantle", a concept which might be a possible solution for the energy exhaust problem in a fusion reactor. The concept is compared with the high density divertor. The compatibility to other constraints, limitations and open problems are discussed, in particular the issues of stationarity (feed-back control, thermal instabilities, $q=2$ ), energy confinement, Heexhaust and fuel dilution.
\end{abstract}

\section{Introduction}

With the next step device (ITER) it is expected that the problem of confinement will be solved primarily by the sheer size of the machine. This then allows to obtain $n \tau T$ values sufficiently high for a stationary burning plasma. It turns out that the unsolved problem of energy and particle exhaust is now the outstanding challenge [Rebut 1993][Parker 1993]. A radial power flow of up to $0.5 \mathrm{MW} / \mathrm{m}^{2}$ entering the edge plasma has to be exhausted. If most of this power enters the scrape-off-layer (SOL) convectively, then the divertor has to withstand power loads of up to 1000 $\mathrm{MW} / \mathrm{m}^{2}$ flowing along the fieidlines. Even with optimistic assumptions about field line expansion and inclined target plates, the peak load at the divertor plate will not be much lower than $50 \mathrm{MW} / \mathrm{m}^{2}$. This is far too high for stationary operation. A reduction of the power load to the target plates by about a factor of 10 is indispensable. To achieve this a substantial fraction of the power must be distributed on other parts of the wall. Two potential scenarios are discussed (figure 1).

1) The "high density divertor" has been proposed [Watkins and Rebut 1992] as a new concept. The key idea is that charge exchange and ionization processes of deuterium/tritium serve as mechanisms for energy dissipation on the divertor walls.

2) Energy dissipation on the whole vessel wall can be

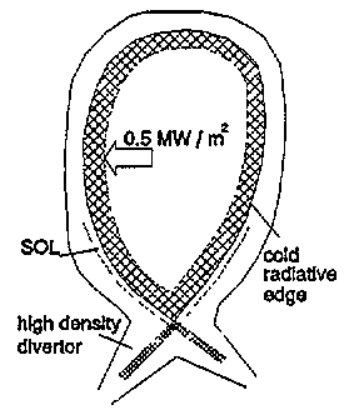

Figure 1 Energy exhaust scenarios

achieved by line radiation from impurities located at the boundary of the bulk plasma, primarily inside the separatrix; this is the basis for the concept of the "cold radiative plasma mantle"; e.g. [Gibson and Watkins 1977, Lackner 1984]. 


\section{B168 U Samm et al}

Unfortunately, up to now the feasibility of the high density divertor has not been shown. Moreover, recent studies even show that probably not more than $35 \%$ of the power can be dissipated inside the divertor by hydrogen processes alone [Stangeby 1993][Vlases 1993]. Therefore, additional power dissipation by radiation from impurities appears to be necessary. According to experimental results obtained so far the radiated power in a divertor can be raised to about $50 \%$ of the total power if impurities are involved [Janeschitz 1992][Shimada 1990][Petrie 1992].

The high electron density foreseen in the divertor is beneficial for impurity radiation, as is evident from an expression for the total radiated power

$$
P_{\text {rad }}=\left\langle\mathrm{n}_{e}\right\rangle\left\langle\mathrm{n}_{\mathrm{i}}\right\rangle \mathrm{L}_{\text {eff }} \mathrm{V} \text {, }
$$

where $\left\langle\mathrm{n}_{\mathrm{e}}\right\rangle$ and $\left\langle\mathrm{n}_{\mathrm{i}}>\right.$ are the electron and impurity ion densities, $\mathrm{L}_{\mathrm{eff}}$ is the effective cooling rate for a given impurity (mainly depending on the electron temperature $T_{e}$ ) and $V$ is the effective volume of the radiating zone. The volume of the radiating zone is limited firstly by the extent of the divertor, and may be even smaller in cases where the radiating ions are not distributed all over the whole divertor volume. Considering the possible volume of the cold radiating plasma boundary inside the separatrix, in particular when comparing the SOL-thickness of about $1 \mathrm{~cm}$ with the typical radial extent of a radiating boundary of $20 \mathrm{~cm}$, it is evident that the limitation in volume is the main disadvantage for impurity radiation in a divertor (see figure 1).

There is no significant difference in the cooling rates for the divertor and the radiating boundary, as can be seen in figure 2, where $L\left(T_{e}\right)$, obtained from model calculations [Tokar" 1992], is shown for neon particles transported along the minor radius in the plasma boundary typical for TEXTOR and parallel to the field in an ITER-like divertor plasma. For comparison the cooling rates for coronal equilibrium [Jensen and Post 1977] are also shown. It turns out that transport is the important source of difference. In the case of radial transport the radiation can be extended to regions with much higher $T_{e}$ values, thus expanding the effective volume available for radiation.

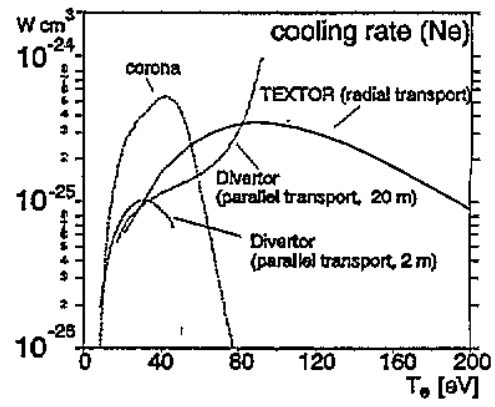

Figure 2 Calculated cooling rates for neon. The spatial coordinates (radius, magnetic field) have been transformed into local $\mathrm{T}_{0^{-}}$ values.

This effect is an important element in the concept of the "cold radiative boundary", the feasibility of which has been demonstrated with experiments on TEXTOR [Samm 1991,1992a,b]. A high radiation level exceeding even $90 \%$ of the total heating power $(\approx 4 \mathrm{MW}$ ) has been achieved for quasi-stationary conditions by feed-back injection of neon. The presentation of these data, including also recent experiments where silicon has been employed for edge cooling, is the main aim of this paper. The possibilities and limitations of this concept, in particular the issue of stationarity and impurity transport, will be discussed.

\section{Experiment}

TEXTOR is a medium-size tokamak with a circular plasma having a major radius of $R=1.75 \mathrm{~m}$ and a minor radius of $a=0.46 \mathrm{~m}$. The standard values are for the magnetic field $\mathrm{B}_{\mathrm{T}}=2.25 \mathrm{~T}$ and for the plasma current $I_{P}=350 \mathrm{kA}$. The toroidal belt limiter ALT-II is used as a pump limiter. All limiter blades are made of graphite and the vessel wall is either boronized or siliconized [Winter 1993]. The working gas is deuterium or hydrogen. Auxiliary heating with neutral beam coinjection (NBI co) of $\mathrm{H}$ or D (1.4 MW) and ion-cyclotron resonance heating ICRH (2 MW) 
together with ohmic heating $(0.3 \mathrm{MW})$ provide a total heating power of $P_{\text {tot }} \approx 3.7 \mathrm{MW}$. This corresponds to an average power density of $\approx 0.5 \mathrm{MW} / \mathrm{m}^{3}$ and a radial power flow to the edge of $\approx 0.11 \mathrm{MW} / \mathrm{m}^{2}$. Injection of additional impurities is obtained by employing gaseous species like neon or di-silane $\left(\mathrm{Si}_{2} \mathrm{H}_{6}\right)$ via a gas puffing system with a fast piezo-electric valve.

The radial profile of the total radiation is measured by means of bolometry. Emission spectroscopy measures the intensity of various lines at the limiters, e.g. $\mathrm{H}_{\alpha}, \mathrm{CI}, \mathrm{O}$ I, Ne I, Ne MI, thus providing information about particle fluxes emitted from these limiters. Atomic beam diagnostics employing $\mathrm{He}-, \mathrm{Li}-$ or $\mathrm{C}$-atoms allow one to determine the profiles of electron density and temperature at the plasma boundary [Schweer 1992][Pospieszczyk 1989]. Information on the impurity concentration in the centre can be deduced from the measurements of soft X-ray emission, from charge exchange spectroscopy and from the electrical conductivity in the plasma centre.

\section{The "cold radiative boundary" - experimental evidence}

The radial extent of the radiative layer has to be compatible with the fusion processes in the bulk plasma. It can easily be shown that, according to the $n_{\mathrm{e}}-$ and $T_{\mathrm{e}^{-}}$ profiles expected for ITER [Rebut 1993], essentially $100 \%$ of the fusion power will be generated within $80 \%$ of the minor radius; thus for $r / a>0.8$ high impurity concentrations and low plasma temperatures do not affect the fusion yield, provided that selective cooling of the edge region is possible.

The experimental data obtained on TEXTOR with neon or with silicon as radiating impurities indeed prove that the edge can be cooled without changing the plasma temperature in the core. For example, two cases are compared in figure 3 , showing the $T_{e}$-profiles for a low $(40 \%)$ and a high radiation $(85 \%)$ level $\left(\mathrm{P}_{\mathrm{rad}} / \mathrm{P}_{\text {heat }}\right)$ obtained by neon injection in a siliconized machine. The line-averaged electron density $\bar{n}_{e}$ is kept constant. $T_{e}$ in the centre remains clearly constant (or even increases slightly), whereas $T_{e}$ at the edge is significantly reduced, in accordance with the reduced convective power flow to the limiters. The drop in $T_{e}$ starts at a radius of $r / a \approx 0.75$, which coincides with the location of the radiative layer (figure 4).

Only impurity species with a sufficiently low mass
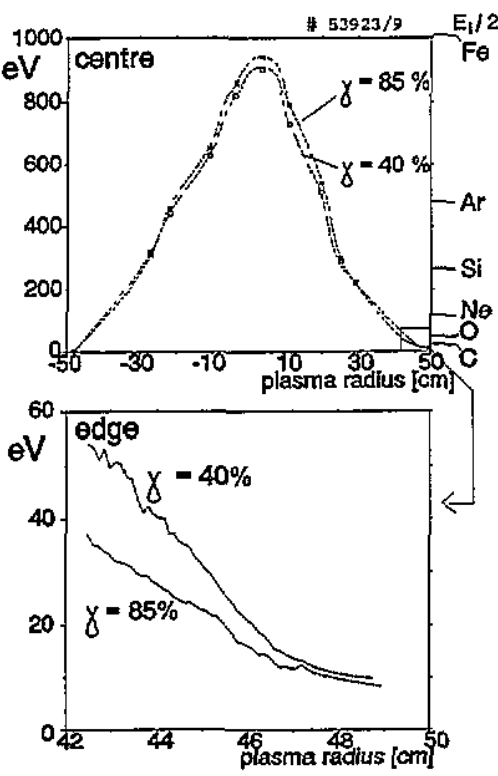

Figure 3 Electron temperature profiles with and without neon injection; $\mathrm{P}_{\text {tot }}=1.6 \mathrm{MW}, \gamma=\mathrm{P}_{\mathrm{rad}} / \mathrm{P}_{\text {heat }}$, $\overline{\mathrm{n}}_{\mathrm{e}}=4.510^{13} \mathrm{~cm}^{-3}$

number fulfill the requirement that line radiation be restricted to the plasma boundary. According to a simple rule, the extent of this radiative edge is essentially limited by the ionization of the $\mathrm{Li}-$ like ions. This ionization occurs at $\mathrm{T}_{\mathrm{e}} \approx \mathrm{E}_{\mathrm{i}} / 2$, where $\mathrm{E}_{\mathrm{i}}$ is the ionization energy of the Li-like stage. The sharp increase of the ionization energy from the Li-like to the He-like stage plays an important role for suppression of radiation further inside the bulk plasma. The location of $T_{e}=E_{j} / 2$ for various species is indicated in figure 3 . It can be seen that Ar is already critical, whereas $\mathrm{Fe}$ clearly is unacceptable for the $T_{e}$-profiles displayed in figure 3 . Of course, under ITER conditions, elements with higher $Z$ would be acceptable and elements like neon would radiate at much larger radii $r / a$ than those found in TEXTOR. 
An important question is, whether these low$Z$ elements, which provide selective edge cooling, are able to radiate on a sufficiently high power level, since it is known that the radiated energy per impurity particle increases strongly with $Z$ (approximately as $Z^{3}$ ). The results obtained on TEXTOR prove that already with light species like $\mathrm{Ne}$ or $\mathrm{Si}$, a significant fraction of the total power can be radiated (figure 5). At a medium lineaveraged electron density $\left(\bar{n}_{\mathrm{e}}=4.10^{13} \mathrm{~cm}^{-3}\right)$ and a medium heating power level (1.6 MW) the radiation level can go up to $98 \%$ with injected neon. The discharges are stable and quasi-stationary with a flat top of $\approx 1$ s (see figure 6a).

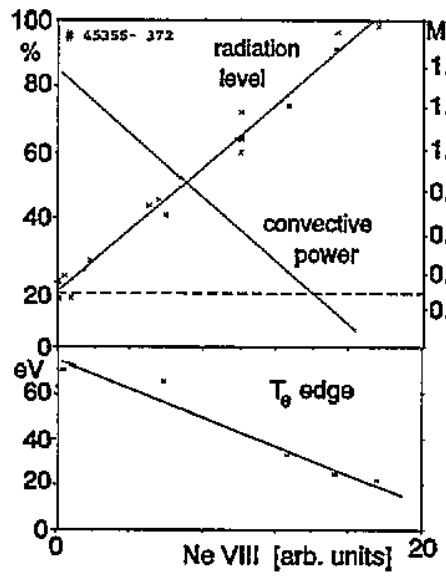

Figure 5 Radiation level, convective power to limiter and edge $\mathrm{T}_{\mathrm{e}}$ as a function of the amount of injected neon.

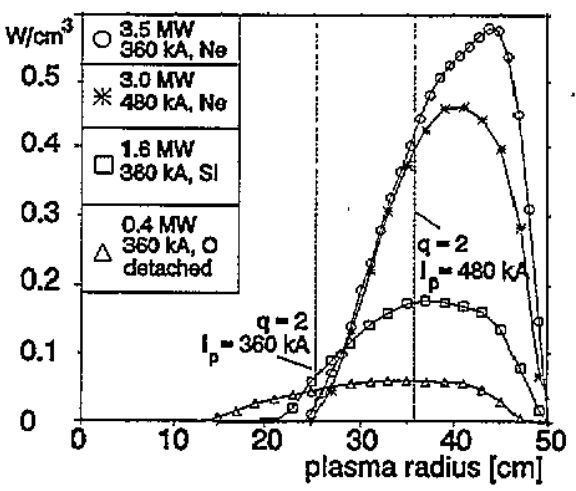

Figure 4 Radial radiation profiles for various conditions of heating power, plasma current and impurities; the location of the $q=2$ surface is indicated, all cases $\gamma>75 \%$.

Silicon has also been employed as a radiating impurity, showing a behaviour in general similar to that of neon, in particular with respect to the power level and the extent of the radiating zone (figures 4 and 8 ). The source of $\mathrm{Si}$ was sputtering on limiter and wall after siliconization [Winter 1993] and/or gas puffing of di-silane $\left(\mathrm{Si}_{2} \mathrm{H}_{6}\right)$. Compared to neon, the possibility to control the level of silicon in the plasma is quite different from that of neon, since Si sticks to the wall - in contrast to the recycling neon (see next section). Also the combination of neon injection with a siliconized wall has been applied successfully.

Neon radiates in TEXTOR at least about $\mathrm{E}_{\mathrm{rad}}=6 \mathrm{keV}$ per injected particle. $\mathrm{E}_{\mathrm{rad}}$ is the "radiation potential" representing the energy a particle radiates during its dwell time in the plasma [Samm 1992b]. $E_{\mathrm{rad}}$ can significantly increase with plasma cooling. Measurements show that in a cold plasma boundary $(\mathrm{Te}(\mathrm{a}) \approx 10 \mathrm{eV}) \mathrm{E}_{\mathrm{rad}}$ can reach values of up to $30 \mathrm{keV}$ (e.g. the discharge shown in figure $6 \mathrm{~b}$ ). To radiate a power of $4 \mathrm{MW}$ a Ne fuelling rate of $\mathrm{F}_{\mathrm{Ne}}=8.10^{20} \mathrm{~s}^{-1}$ is needed. The values for $\mathrm{Si}$ are similar. A peak radiation of $600 \mathrm{~kW} / \mathrm{m}^{3}$ has been obtained in TEXTOR. 

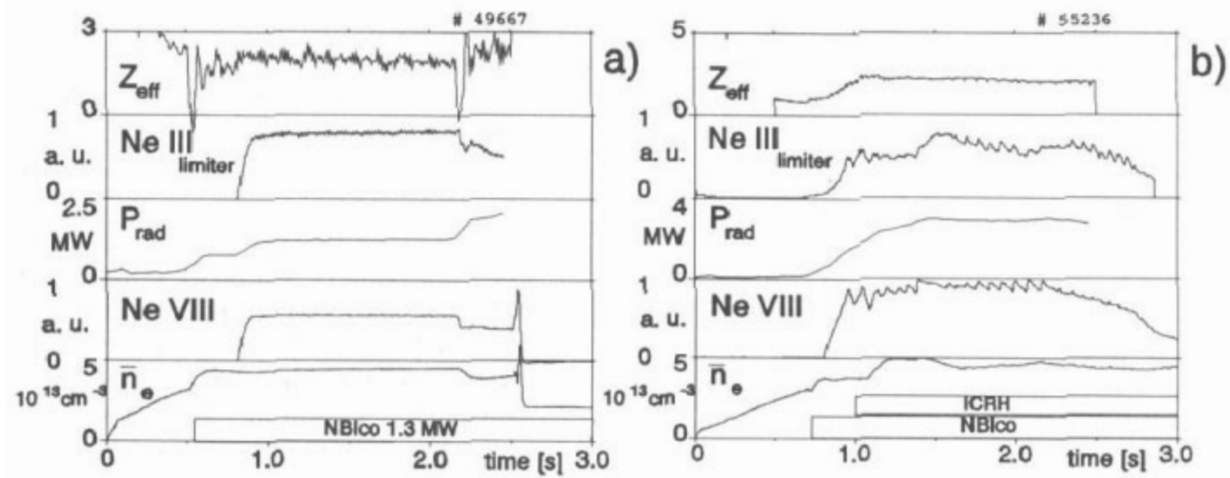

Figure 6 Time traces of discharges with a high radiation level:

a) $P_{\text {heat }}=1.6 \mathrm{MW}, \gamma=80 \%$, b) $P_{\text {heat }}=3.5 \mathrm{MW}, \gamma=85 \%$

We conclude that the results which have been presented here may serve as a proof of feasibility showing that low $Z$ elements - in the range of neon or silicon - are valuable candidates to provide the necessary energy dissipation on the wall. In the following sections the limitations and open questions of the concept will be discussed.

\section{Stationarity}

\section{feed-back control of the impurity level}

Quasi-stationary operation of discharges at a radiation level close to $100 \%$ is only possible with a very well controlled impurity level. After the first experiments on TEXTOR with neon gas puffing, it became evident that a feed-back system is indispensable [Samm 1991]. A feed-back system can only work with a source and a sink for the impurities to be controlled. In case of neon the sink is given by the pump limiter (ALTII). With the pumps activated, the effective removal time $\tau_{P}^{*}$ for $\mathrm{Ne}$ is about $0.35 \mathrm{~s}$. The feed-back system developed on TEXTOR is using a Ne VIII line as the control variable. This line adequately represents the total radiation from neon. The system allows the pre-programming of a given radiation level. an example of which is shown in figure 7 . Note that the lines of Ne I (neon entering the plasma) and $\mathrm{Ne}$ VIII (neon in the bulk) are varying similarly in time, thus indicating that no accumulation of Ne occurs during the flat top of the radiation.
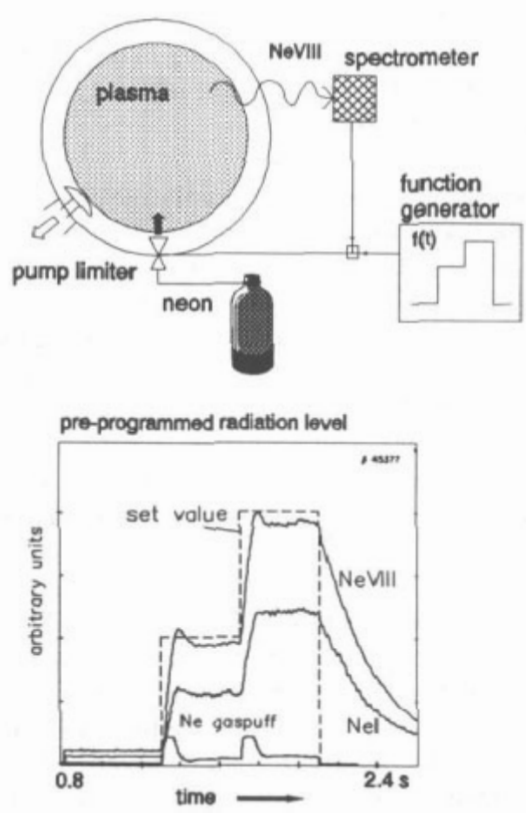

Figure 7 The neon feed-back system on TEXTOR. 


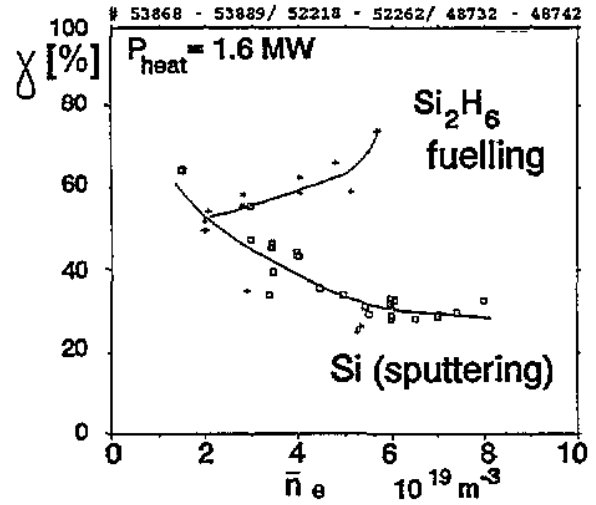

Figure 8 Radiation level $\gamma$ as a function of the line averaged electron density for a siliconized wall with/without di-silane fuelling

neon other means for silicon injection have to be developed.
The control of silicon is more difficult. In a siliconized machine the intrinsic source for $\mathrm{Si}$ is sputtering, a process which depends strongly on the plasma edge temperature. It is therefore possible that with increasing electron density the silicon radiation decreases due to a drop of the sputtering yield (see figure 8)[Unterberg 1993]. Additional radiation from $\mathrm{Si}$ can be obtained e.g. by $\mathrm{Si}_{2} \mathrm{H}_{6}$ puffing, but the possible variation is limited due to the unavoidable density increase arising from the injection of molecules containing hydrogen. The maximum radiation level obtained so far was about $\gamma=75 \%$ with full di-silane fuelling (figure 8). To increase the radiated power on the same level as for

\section{thermal instabilities}

The phenomenon of a radiative condensation instability may occur in a cold plasma, when the local heat flux balance is strongly affected by increasing energy losses from radiation with decreasing $T_{e}$. In this case usually another quasi-stable regime develops with low $T_{e}$ and a high local radiation level. The relevant parameter is the ratio of $P_{r a d} / P_{\text {heat }}$ in a global and in a local sense. The radiative condensation occurs when this ratio exceeds a certain value. How this is achieved can be demonstrated by many different examples obtained in TEXTOR : e.g. auxiliary power switch off, density rise, impurity injection, current ramp down or particle flow increase due to a plasma shift.

The local appearance of this radiative condensation can be quite different. If the ratio $P_{\text {rad }} / P_{\text {heat }}$ is increasing uniformly within the plasma boundary, then we observe a plasma detachment [Samm 1987]; i.e. a poloidally symmetric radiating belt in a plasma with a significantly reduced minor radius. But at high heating powers, leading to high- $\beta$ plasmas with a pronounced Shafranov-shift, the radial heat flux exhibits strong poloidal asymmetries with the consequence of a poloidally asymmetric appearance of this radiative condensation; this is called a MARFE [Lipschultz 1989]. Inside a MARFE the electron density easily exceeds $10^{14} \mathrm{~cm}^{-3}$ and $\mathrm{T}_{\mathrm{e}}$ drops well below $5 \mathrm{eV}$ [Sergienko 1993]. The total power radiated from a MARFE is in the order of $20 \%-50 \%$ of the total input power. It is important to note that radiation from a MARFE may lead to significant peak loads on the wall in the vicinity of the MARFE.

Furthermore, both phenomena, detachment and MARFEs, have disadvantages. Detachment is linked to reduced energy confinement $(\approx 30 \%)$ and MARFEs are normally not stationary, but move in the poloidal direction and in most cases finally lead to a major disruption. Several approaches for the theoretical understanding of MARFEs have been made, but are far from sufficient to explain and predict all phenomena [Neuhauser 1986][Nedospasov 1988]. Experimentally, MARFEs can be avoided by operating not too close to the density limit. E.g. if in TEXTOR under certain conditions the density limit is at about $\bar{n}_{\mathrm{e}}=5.510^{13} \mathrm{~cm}^{-3}$, operation at $\bar{n}_{e}=5.10^{13} \mathrm{~cm}^{-3}$ provides enough margin to allow a high radiation level $(90 \%)$ without the generation of MARFEs. 


\section{MHD instabilities}

From low power discharges, where detachment occurs at a high radiation level, we know that operation at low q normally gives rise to major disruptions [Samm 1987]. It was argued, that the local coincidence of the radiating layer and the $q=2$ surface may trigger MHD instabilities. In contrast to this, experiments performed recentiy at a high power level $(3.5 \mathrm{MW})$ and $q(a)=2.7$ $\left(\mathrm{I}_{\mathrm{P}}=480 \mathrm{kA}\right)$ showed that stationary conditions with a radiation level of at least $80 \%$ are possible, although the $\mathrm{q}=2$ surface is located inside the radiating layer (figure 4). An important difference could be, that with detached plasmas and increasing plasma current, the $q=2$ surface moves to the outside of the radiation maximum and may then be the cause of a disruption (figure 4).

\section{Compatibility with energy confinement}

At medium heating power and with increasing radiation by neon or silicon in jection, the global energy confinement is at least maintained. Operation at high heating power ( $>2.5 \mathrm{MW})$ even reveals an increase in energy confinement time with radiative edge cooling [Messiaen 1993]. This effect becomes more pronounced the higher the average electron density. It is speculated that a kind of profile shaping by radiation leading to steeper $T_{e}$-profiles at the edge may play an important role in this context. Furthermore, it should be noted that on an absolute scale (scaling law ITER L89-P) the energy confinement is improved by factors up to 1.7 depending on density and radiation level [Ongena 1992 and 1993]. For example, the discharge presented in figure 6b has an enhancement factor of 1.5.

\section{He-exhaust}

Efficient He removal (ash exhaust) is an important requirement for a fusion reactor [Reiter 1991]. Experiments on TEXTOR demonstrate that efficient He pumping with the pump limiter ALT-II [Hillis 1990] is still possible in a plasma with a cold radiative edge [Samm 1992a]. The He transport can be strongly affected by friction with the background plasma; it is possible that in a cold plasma the Mach-profiles of plasma flow towards the limiter are changing in such a way that higher velocities at the scoop entrance of the pump limiter lead to a more efficient flush back of He-ions than in a hot edge plasma. As a consequence, efficient removal of He is maintained, in spite of the fact that the He confinement in the bulk plasma is increasing.

\section{Impurity transport and fuel dilution}

The crucial limitation for the application of radiative edge cooling by impurities is given by fuel dilution in the bulk plasma, thus depending on the impurity transport - unfortunately a problem we know the least about compared to all the critical issues discussed so far. Optimism may be gained from the experimental results obtained on TEXTOR, where even for the highest radiation levels obtained, the central impurity level never exceeded $1 \%$ of $n_{e}$. Although this would be an acceptable value for ITER, a reliable extrapolation to ITER is impossible. However, a remarkable result is that impurities behave differently compared to the background plasma, as can be seen most clearly from the results obtained with neon. Based on the fact that neon is fully recycling (not stored in the wall) and on the knowledge of the total amount of neon in the discharge obtained from the calibrated gas-injection system, we may try to compare different transport schemes with the measured neon concentration in the centre (soft X-ray emission) and at the separatrix. The latter one is derived from the measured particle fluxes of neon and deuterium flowing to and emitted from the limiters (emission spectroscopy; $\mathrm{Ne} I, \mathrm{H}_{\alpha}$ ) based on the assumptions of an average charge for neon of $z=4$ and equal flow velocities of neon ions and deuterons in the SOL. The different profiles (summed over all charge states) are shown in figure 9 , where neon is distributed a) like the electrons, b) according to a diffusion model 


\section{B174 U Samm et al}

[Engelhardt 1982] generally yielding a flat profile beyond the ionization zone and c) as a hollow profile. The measured concentration in the centre yields $0.13 \%$. Compared to this value, the distribution a) is far too high (factor 3 ) and the flat distribution is still somewhat larger. Agreement is found with the hollow profile, obtained by adjusting the level to the measured data and by fitting the width of the edge part $(5 \mathrm{~cm})$. If this behaviour of light impurities were maintained also under ITER conditions, it would be of great importance for the purpose of edge cooling. The underlying transport mechanisms responsible for this effect are not clear, possibly a kind of temperature screening may play a role. Further indications for such behavior of light impurities are also found with silicon and from transport studies with carbon [Claassen 1990].

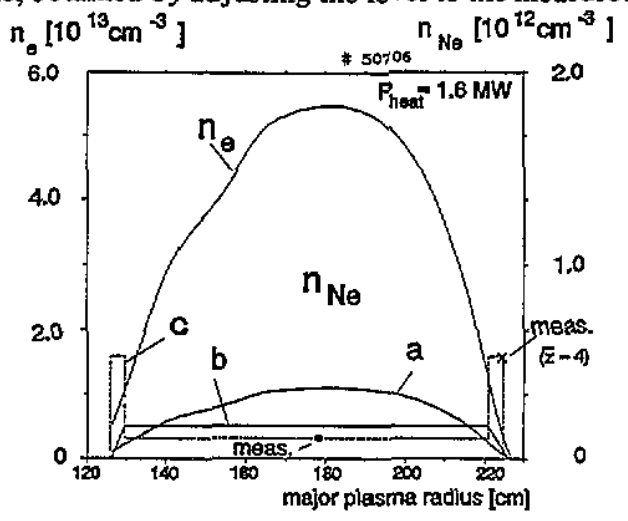

\section{Conclusions}

Figure 9 Radial electron-and neon-density distribution. Different transport schemes for neon : a) like electrons, b) diffusion model, c) hollow profile.

The TEXTOR data presented here may serve as a proof of principle for the feasibility of the "cold radiating plasma mantle" as a concept for energy exhaust in a fusion reactor. This concept could be used in combination with the high density divertor - with the benefit of relieving the divertor - or as a stand-alone solution, in which case even a fusion reactor having limiters and no divertor becomes conceivable. No reliable prediction and extrapolation e.g. to ITER is possible. However, the TEXTOR data demonstrate that it seems likely to overcome the problems of impurity control, thermal instabilities, $M H D$-instabilities, energy confinement and He-exhaust by choosing a suitable combination of parameters, like e.g. the impurity species used for radiation (neon, silicon), the average electron density and wall materials (surface layers) compatible with the whole concept. Ultimately, the maximum radiation level possible in ITER will be limited by fuel dilution in the bulk plasma, thus the impurity transport is the crucial process on which future research has to concentrate on. TEXTOR data indicate that conditions exist, where the radial distribution of impurities is flat or even hollow, in contrast to the peaked profiles of the electrons and deuterons, thus generating a high impurity level at the edge and a low one in the centre.

\section{Acknowledgements}

The TEXTOR team is gratefully acknowledged for providing excellent experimental conditions. We wish to thank G. Esser, A. Krämer-Flecken, H.R. Koslowski, A. Messiaen, J. Ongena, G. Telesca, J. Schlüter, H. Soltwisch, G. Waidmann and J. Winter for providing valuable data and for some clarifying discussions.

\section{References}

Claassen H.A et al. $1990 \mathrm{~J}$. Nucl. Mater. 176-177 398

Engelhardt W et al. 1982 J. Nucl. Mater. 111-112 337

Gibson A and Watkins M.L. 1977 Contr. Fusion and Plasma Physics 131

Hillis D.L et al. 1990 Phys. Rev. Lett. 65192382

Janeschitz $\mathrm{G}$ et al. 1992 in Plasma Physics and Controlled Nuclear Fusion Research 1 (Proc. 14th Int. Conf. Würzburg 1992, IAEA Vienna 1993)

Jensen R.V and Post DE. 1977 Atomic Data and Nuclear Data Tables 205

Lackner K, et al. 1984 Plasma Physics and Controlled Fusion $261 \mathrm{~A} 105$ 
Lipschultz B 1989 J. Nucl. Mater. 145-147 15

Messiaen A, et aI. 1993 submitted to Nucl. Fus. Lett.

Nedospasov A.V, et.al. 1988 Contr. to Plasma Physics $284 / 5453$

Neuhauser $\mathrm{J}$ et al. 1986 Nucl. Fus. 2612

Ongena J et al. 1992 in Plasma Physics and Controlled Nuclear Fusion Research 1725 (Proc. 14th Int. Conf. Würzburg 1992, IAEA Vienna 1993)

1993 Proceedings of the 20th Europ. Conf. on Plasma Physics and Controlled Fusion, Lisbon I 127

Parker R 1993 Proceedings of the 20th Europ. Conf. on Plasma Physics and

Controlled Fusion, Lisbon, to be published in Plasma Physics and Controlled Fusion

Petrie T.W et al. 1992 J. Nucl. Mater. 196-198 849

Pospieszczyk A et al. $1989 \mathrm{~J}$. Nucl. Mater. 162-164 574

Rebut P.H et al. 1993 JET Report JET-P(93)06

Reiter D et al. 1991 Plasma Physics and Controlled Fusion 33131579

Samm U et al. $1987 \mathrm{KFA}$ Jül-Report 2123

1991 Proceedings of the I8th Europ. Conf. on Plasma Physics and Controlled Fusion, Berlin 3157 1992a J. Nucl. Mater. 196-198 633 1992b in Plasma Physics and Controlled Nuclear Fusion Research 1309 (Proc. 14th Int. Conf. Würzburg 1992, IAEA Vienna 1993)

Schweer B et al. $1992 \mathrm{~J}$. Nucl. Mater. 196-198 174

Sergienko G et al. 1993 Proceedings of the 20th Europ. Conf. on Plasma Physics and Controlled Fusion, Lisbon II 667

Shimada M et al. $1990 \mathrm{~J}$. Nucl. Mater. 176-177 122

Stangeby P.C 1993 JET report JET-P(93)13, submitted to Nucl. Fus. Lett.

Tokar' M 1992 KFA Jül-Report 2588

Unterberg B et al. 1993 Proceedings of the 20th Europ. Conf. on Plasma Physics and Controlled Fusion, Lisbon II 663

Watkins M.L and Rebut P.H 1992 Proceedings of the 19th Europ. Conf. on Plasma Physics and Controlled Fusion, Innsbruck II 731

Vlases G et al. 1993 Proceedings of the 20th Europ. Conf. on Plasma Physics and Controlled Fusion, Lisbon, to be published in Plasma Physics and Controlled Fusion

Winter J et al. 1993 Proceedings of the 20th Europ. Conf. on Plasma Physics and Controlled Fusion, Lisbon I 279 